\title{
Weibull-Normal Distribution and its Applications
}

\author{
Felix Famoye ${ }^{1, \star}$, Eno Akarawak ${ }^{2}$, Matthew Ekum ${ }^{3}$ \\ ${ }^{1}$ Department of Mathematics, Central Michigan University, Mt. Pleasant, Michigan, USA \\ ${ }^{2}$ Department of Mathematics, University of Lagos, Akoka-Yaba, Lagos, Nigeria \\ ${ }^{3}$ Department of Mathematics \& Statistics, Lagos State Polytechnic, Ikorodu, Lagos, Nigeria
}

\section{ARTICLE INFO}

\section{Article History}

Received 2 March 2016

Accepted 19 July 2017

\section{Keywords}

$\mathrm{T}-\mathrm{R}\{\mathrm{Y}\}$ framework

Bimodal

Hazard function

Estimation

\section{Mathematics Subject}

Classification

62E15, 62F10, $62 \mathrm{P} 99$

\section{ABSTRACT}

In this paper, a Weibull-normal distribution, based on the standard quantile function of log-logistic distribution, is defined and studied. Some properties of the probability distribution are discussed. The Weibull-normal distribution is found to be unimodal or bimodal. The distribution can be right skewed or left skewed. The method of maximum likelihood estimation is suggested to estimate the parameters of the distribution. Three numerical data sets are used to illustrate the applications of the Weibullnormal distribution.

\section{INTRODUCTION}

The theory of statistical distributions continues to be a strong area of research. A lot has been done on the univariate distributions and in particular, the continuous univariate distributions. For a review of methods of generating statistical distributions in the recent decades, the reader is referred to Ref. [1]. In this reference, a chronological reference to how the beta-generated family, the Kumaraswamy generated (Kum-generated for short) family and the generalized beta-generated family led to the $T$ - $X$ family of distributions. Subsequently, we have the $T-R\{Y\}$ family introduced by Ref. [2], with a unified notation provided in Ref. [3].

The beta-generated family of distributions was introduced by Ref. [4]. The cumulative distribution function (CDF) of the family is given by

$$
G(x)=\int_{0}^{F(x)} b(t) d t
$$

where $b(t)$ is the probability density function (PDF) of the beta distribution and the CDF $F(x)$ is that of any discrete or continuous random variable $X$. The associated PDF to Eq. (1) is

$$
g(x)=\frac{\Gamma(\alpha+\beta)}{\Gamma(\alpha) \Gamma(\beta)}[F(x)]^{\alpha-1}[1-F(x)]^{\beta-1} f(x), \alpha>0, \beta>0 .
$$

When $F(x)$ is the CDF of the normal distribution, the result in Eq. (2) defines the beta-normal (BN) distribution. When $\alpha$ and $\beta$ are both integers, the result in Eq. (2) is the distribution of the $\alpha^{\text {th }}$ order statistic from a random sample of size $(\alpha+\beta-1)$. The BN distribution can be unimodal or bimodal. It can be skewed to the left, or skewed to the right, or symmetric. The BN distribution has been applied to fit a variety of data sets (Ref. [5]).

The beta-generated family of distributions was extended (Refs. [6] and [7]) to the Kumaraswamy-generated family of distributions by replacing the beta distribution with Kumaraswamy distribution. The PDF of the Kumaraswamy distribution (Ref. [8]) is given by $f(t)=$ $\alpha \beta t^{\alpha-1}\left(1-t^{\alpha}\right)^{\beta-1}$ for $0<t<1$. The CDF of the Kumaraswamy-generated family of distributions is given by

$$
G(x)=1-\left(1-[F(x)]^{\alpha}\right)^{\beta} .
$$

*Corresponding author. Email: felix.famoye@cmich.edu 
Another extension of the beta-generated family of distributions, the generalized beta-generated family of distributions, was proposed by Ref. [9]. These authors used the generalized beta of type I distribution to replace the beta distribution.

The $T-X(W)$ family of distributions, which extended the beta-generated, Kumaraswamy-generated, and generalized beta-generated families, was introduced by Ref. [10]. The CDF of the family is given by

$$
G(x)=\int_{a}^{W(F(x))} r(t) d t
$$

where $r(t)$ is the PDF of a random variable $T$ with support $(a, b),-\infty \leq a<b \leq \infty$. The function $W(F(x))$ is monotonic and absolutely continuous. The $T-X(W)$ was modified as $T-R\{Y\}$ by Ref. [2] by taking the $W(F(x))$ to be the quantile function of a random variable $Y$. By using the unified notation in Ref. [3], we have $T, R$ and $Y$ as random variables with $\operatorname{CDF} F_{Z}(x)=P(Z \leq x)$ for $Z=T, R$ and $Y$. The corresponding quantile functions for the three random variables are $Q_{T}(p), Q_{R}(p)$ and $Q_{Y}(p)$ where $Q_{Z}(p)=\inf \left\{z: F_{Z}(z) \geq p\right\}$ for $0<p<1$. When the densities exist, we denote them by $f_{T}(x), f_{R}(x)$ and $f_{Y}(x)$. It is assumed that the random variables $T \in(a, b)$ and $Y \in(c, d)$, for $-\infty \leq a<b \leq \infty$ and $-\infty \leq c<d \leq \infty$. By using the unified notation, the CDF of a random variable $X$ that belongs to a $T$ - $R\{Y\}$ family of distributions is

$$
F_{X}(x)=\int_{a}^{Q_{Y}\left(F_{R}(x)\right)} f_{T}(t) d t=P\left(T \leq Q_{Y}\left(F_{R}(x)\right)=F_{T}\left\{Q_{Y}\left(F_{R}(x)\right)\right\}\right.
$$

When $R$ is a normal random variable, we have $F_{R}(x)=\Phi(x)$, the CDF of normal distribution. Hence, we obtain

$$
F_{X}(x)=\int_{-\infty}^{Q_{Y}(\Phi(x))} f_{T}(t) d t=F_{T}\left\{Q_{Y}(\Phi(x))\right\}
$$

as the $T$-normal $\{Y\}$ family of distributions studied by Ref. [3].

In Section 2, we define the Weibull-normal\{log-logistic\} distribution. In Section 3, we provide some properties, including the modes and the Shannon entropy. The method of maximum likelihood estimation is proposed for estimating the distribution parameters in Section 4. A simulation study in Section 5 is used to evaluate the performance of the maximum likelihood estimation method. In Section 6, we illustrate the applications of the distribution with three numerical real life data sets and the results are compared with other distributions. We provide a summary in Section 7.

\section{WEIBULL-NORMAL\{LOG-LOGISTIC\} DISTRIBUTION}

Suppose a random variable $T$ follows the Weibull distribution with parameters $c$ and $\gamma$. Then, the CDF of $T$ is given by

$F_{T}(x)=1-\exp \left\{-(x / \gamma)^{c}\right\}$, for $x>0$ and $c, \gamma>0$.

For the log-logistic distribution, the quantile function is given by

$Q_{Y}(p)=p /(1-p)$ for $0<p<1$, and so $Q_{Y}(\Phi(x))=\Phi(x) /(1-\Phi(x))$,

where $\Phi(x)$ is the normal CDF with mean $\mu$ and standard deviation $\sigma$. By using the $F_{T}(x)$ and $Q_{Y}(\Phi(x))$ in Eq. (2), the CDF of Weibullnormal $\{\log$-logistic $\}(\mathrm{W}-\mathrm{N}\{\mathrm{LL}\})$ distribution is given as

$$
F_{X}(x)=1-\exp \left\{-\left(\frac{\Phi(x)}{\gamma[1-\Phi(x)]}\right)^{c}\right\}, \text { for }-\infty<x<\infty
$$

On differentiating Eq. (3) with respect to $x$, the PDF of W-N $\{L L\}$ distribution is given as

$$
f_{X}(x)=\frac{c}{\gamma^{c}} \frac{\varphi(x)}{[1-\Phi(x)]^{2}}\left(\frac{\Phi(x)}{1-\Phi(x)}\right)^{c-1} \exp \left\{-\left(\frac{\Phi(x)}{\gamma[1-\Phi(x)]}\right)^{c}\right\} \text { for }-\infty<x<\infty
$$

where $\varphi(x)$ is the normal PDF with mean $\mu$ and standard deviation $\sigma$.

The hazard function of the $\mathrm{W}-\mathrm{N}\{\mathrm{LL}\}$ is given by

$$
h(x)=\frac{f_{X}(x)}{1-F_{X}(x)}=\frac{c}{\gamma^{c}} \frac{\varphi(x)}{[1-\Phi(x)]^{2}}\left(\frac{\Phi(x)}{1-\Phi(x)}\right)^{c-1}
$$




\subsection{Limits of the PDF and Hazard Function of the W-N $\{L L\}$}

As $x \rightarrow \pm \infty$, the $\lim _{x \rightarrow \pm \infty} f_{X}(x)=0$. As $x \rightarrow-\infty$, the $\lim _{x \rightarrow-\infty} h(x)=0$. Also, as $x \rightarrow+\infty$, the $\lim _{x \rightarrow+\infty} h(x)=\infty$. In Figure 1 , we have the PDFs of W-N\{LL\} distribution for various values of $c$ and $\gamma$. These curves show that the distribution can be unimodal or bimodal depending on the values of parameter $c$. Also, the distribution can be skewed to the right or skewed to the left. In Figure 2, we present the corresponding hazard functions for the PDFs in Figure 1. As the limits reveal, the limit of $h(x)$ on the left hand side is 0 and the limit on the right hand side is infinity. For the well-pronounced bimodal cases (when $c$ is very small), the hazard function first increases, then decreases and finally increases without bound.

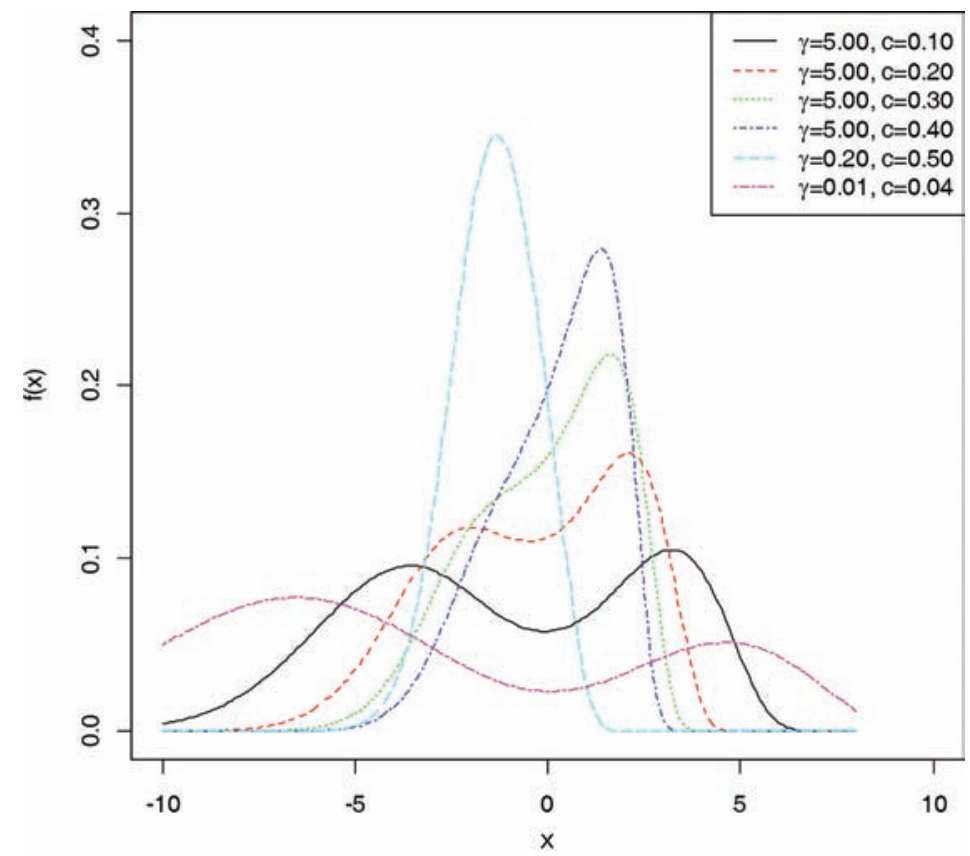

Figure 1 PDFs of W-N $\{\mathrm{LL}\}$ for $\mu=0$ and $\sigma=1$ for various values of $c$ and $\gamma$.

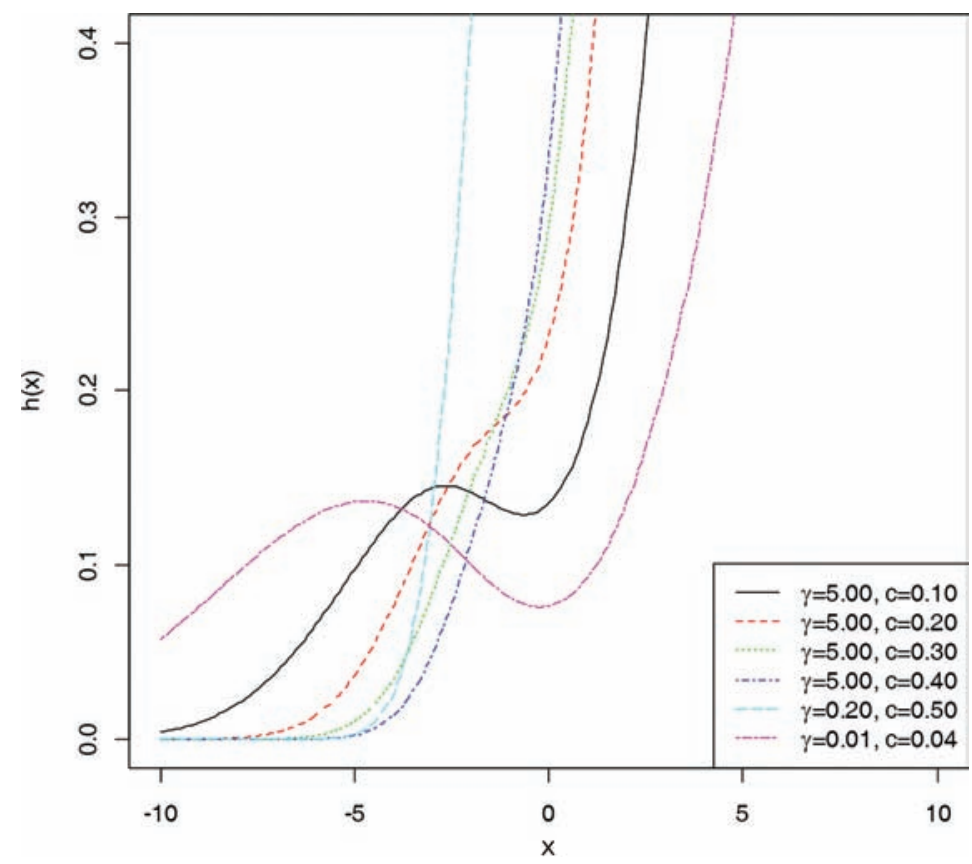

Figure 2 Hazard functions of W-N $\{\mathrm{LL}\}$ for $\mu=0$ and $\sigma=1$ for various values of $c$ and $\gamma$. 


\subsection{Quantile Function}

To find the quantile function of $\mathrm{W}-\mathrm{N}\{\mathrm{LL}\}$, we solve the equation $F_{X}(x)=1-\exp \left\{-\left(\frac{\Phi(x)}{\gamma[1-\Phi(x)]}\right)^{c}\right\}=p$ for $x$. Thus, we obtain the quantile function as

$$
x=Q_{X}(p)=\Phi^{-1}\left(\frac{\gamma[-\log (1-p)]^{1 / c}}{1+\gamma[-\log (1-p)]^{1 / c}}\right) .
$$

Observe that the result $\Phi^{-1}(u)$ in Eq. (5) is the quantile function of a normal random variable with mean $\mu$ and standard deviation $\sigma$ evaluated at the value of $u$.

The median of the W-N $\{L L\}$ is given by $\tilde{x}=Q_{X}(0.5)=\Phi^{-1}\left(\gamma[\log (2)]^{1 / c} /\left\{1+\gamma[\log (2)]^{1 / c}\right\}\right)$. To simulate a random variate $X$ from $\mathrm{W}$-N $\{\mathrm{LL}\}$ distribution, we simulate a uniform random variate $p$ from the interval $(0,1)$ and then calculate the right hand side of Eq. (5).

\section{SOME PROPERTIES OF WEIBULL-NORMAL\{LOG-LOGISTIC\} DISTRIBUTION}

In this section, we provide some properties of the $\mathrm{W}-\mathrm{N}\{L L\}$ distribution.

\subsection{Transformations}

Lemma 1. If $X$ follows a $W-N\{L L\}$ distribution with parameters $c, \gamma, \mu$ and $\sigma$, then the random variable $Y=\Phi(X) /[1-\Phi(X)]$, has a Weibull distribution with parameters $c$ and $\gamma$, where $\Phi(x)$ is the CDF of a normal distribution with parameters $\mu$ and $\sigma$.

Proof. By using the transformation method, the result follows.

The above lemma can be used to transform the $\mathrm{W}-\mathrm{N}\{\mathrm{LL}\}$ random variate $X$ into a Weibull random variate $Y$. For example, the starting values for the parameters $c, \gamma, \mu$ and $\sigma$ in a W-N $\{L L\}$ can be obtained by using the moment estimates for normal and Weibull random variates. The $\mathrm{W}-\mathrm{N}\{\mathrm{LL}\}$ is a generalization of the normal distribution. Take the variate as normal random variate and compute the sample mean and sample standard deviation as the initial estimates of $\mu$ and $\sigma$ respectively. Use Lemma 1 to transform the variate $X$ into $Y$, a Weibull random variate. Then obtain the initial estimates of $c$ and $\gamma$ as the corresponding moment estimates.

Lemma 2. Suppose $Y$ has a standard exponential distribution. Suppose $X$ has a normal distribution with mean $\mu$ and standard deviation $\sigma$ such that $\Phi(x) /[1-\Phi(x)]$ follows a Weibull distribution with parameters $c$ and $\gamma$. Then, the distribution of the random variable $X=\Phi^{-1}\left(\gamma y^{1 / c} /\left(1+\gamma y^{1 / c}\right)\right)$ follows the $W-N\{L L\}$ distribution with parameters $c, \gamma, \mu$ and $\sigma$.

Proof. Use the transformation method.

Lemma 3. Suppose Y has a Pareto distribution with $C D F F(y)=1-(k / y)^{\theta}$, for $y \geq k$. Suppose X has a normal distribution with mean $\mu$ and standard deviation $\sigma$ such that $\Phi(x) /[1-\Phi(x)]$ follows a Weibull distribution with parameters $c$ and $\gamma$. Then, the distribution of the random variable $X=\Phi^{-1}\left(\frac{\gamma[-\theta \ln (k / y)]^{1 / c}}{1+\gamma[-\theta \ln (k / y)]^{1 / c}}\right)$ follows the $W-N\{L L\}$ distribution with parameters $c, \gamma, \mu$ and $\sigma$.

Proof. Use the transformation method.

Lemma 4. Suppose $Y$ has a Fréchet distribution with $C D F F(y)=\exp \left(-y^{-\alpha}\right)$, for $y>0$ and $\alpha>0$. Suppose $X$ has a normal distribution with mean $\mu$ and standard deviation $\sigma$ such that $\Phi(x) /[1-\Phi(x)]$ follows a Weibull distribution with parameters $c$ and $\gamma$. Then, the distribution of the random variable $X=\Phi^{-1}\left(\frac{\gamma\left[-\ln \left\{1-\exp \left(-y^{-\alpha}\right)\right\}\right]^{1 / c}}{1+\gamma\left[-\ln \left\{1-\exp \left(-y^{-\alpha}\right)\right\}\right]^{1 / c}}\right)$ follows the $W-N\{L L\}$ distribution with parameters $c, \gamma, \mu$ and $\sigma$.

Proof. Use the transformation method.

Lemma 5. Suppose $Y$ has a Gumbel distribution with CDF $F(y)=\exp \left(-e^{-y}\right)$, for $-\infty<y<\infty$. Suppose X has a normal distribution with mean $\mu$ and standard deviation $\sigma$ such that $\Phi(x) /[1-\Phi(x)]$ follows a Weibull distribution with parameters $c$ and $\gamma$. Then, the distribution of the random variable $X=\Phi^{-1}\left(\frac{\gamma\left[-\ln \left\{1-\exp \left(-e^{-y}\right)\right\}\right]^{1 / c}}{1+\gamma\left[-\ln \left\{1-\exp \left(-e^{-y}\right)\right\}\right]^{1 / c}}\right)$ follows the $W-N\{L L\}$ distribution with parameters $c, \gamma, \mu$ and $\sigma$.

Proof: Use the transformation method. 


\subsection{Modes of W-N\{LL\} Distribution}

The mode(s) of the W-N $\{L L\}$ distribution is given by equating to zero the derivative with respect to $x$ of the PDF $f_{X}(x)$ in Eq. (4). This is given by the equation

$$
x=\mu+\sigma^{2} \frac{\varphi(x)}{1-\Phi(x)}\left\{2+\frac{c-1}{\Phi(x)}+\frac{c}{\Phi(x)}\left(\frac{\Phi(x)}{\gamma[1-\Phi(x)]}\right)^{c}\right\} .
$$

The distribution can be unimodal or bimodal. The parameter region when the distribution is unimodal or bimodal are difficult to obtain analytically. The following results are obtained when the parameter region is investigated numerically on the number of turning points for the function $f_{X}(x)$.

When $c \leq 0.118$, the W-N $\{L L\}$ is bimodal for all values of parameter $\gamma$. When $c>0.336$, the distribution is unimodal for all values of parameter $\gamma$. When $0.119 \leq c \leq 0.336$, the distribution is either unimodal or bimodal. There exist values of parameter $\gamma$ for which the distribution is unimodal while it is bimodal for other values. For example, the distribution is bimodal for $0.61 \leq \gamma \leq 7.96$ when $c=0.23$. By computing the sign changes for $f_{X}^{\prime}(x)$ in the parameter space $0.01 \leq c \leq 1.0$ and $0.01 \leq \gamma \leq 15$, we notice the above situations. A curve is used to estimate the boundaries separating the unimodal region from the bimodal region. The result is presented in Figure 3.

When $0.119 \leq c \leq 0.336$, we obtain the curve $\gamma=1.332-25.29 c+153.1 c^{2}-235.0 c^{3}$ for the lower bound separating the unimodal and bimodal regions in Figure 3. For values of $c$ between $[0.119,0.21)$, the upper bound for the parameter $\gamma$ is very large and they exceed 15 . For example, when $c=0.46$, the upper bound for $\gamma$ is 25.48. For values of $c$ between [0.22, 0.336) and values of $\gamma$ between [1.0, 13.35), we obtain the curve $\gamma=594.3-5968 c+20018 c^{2}-22366 c^{3}$ for the upper bound separating the unimodal and bimodal regions in Figure 3 .

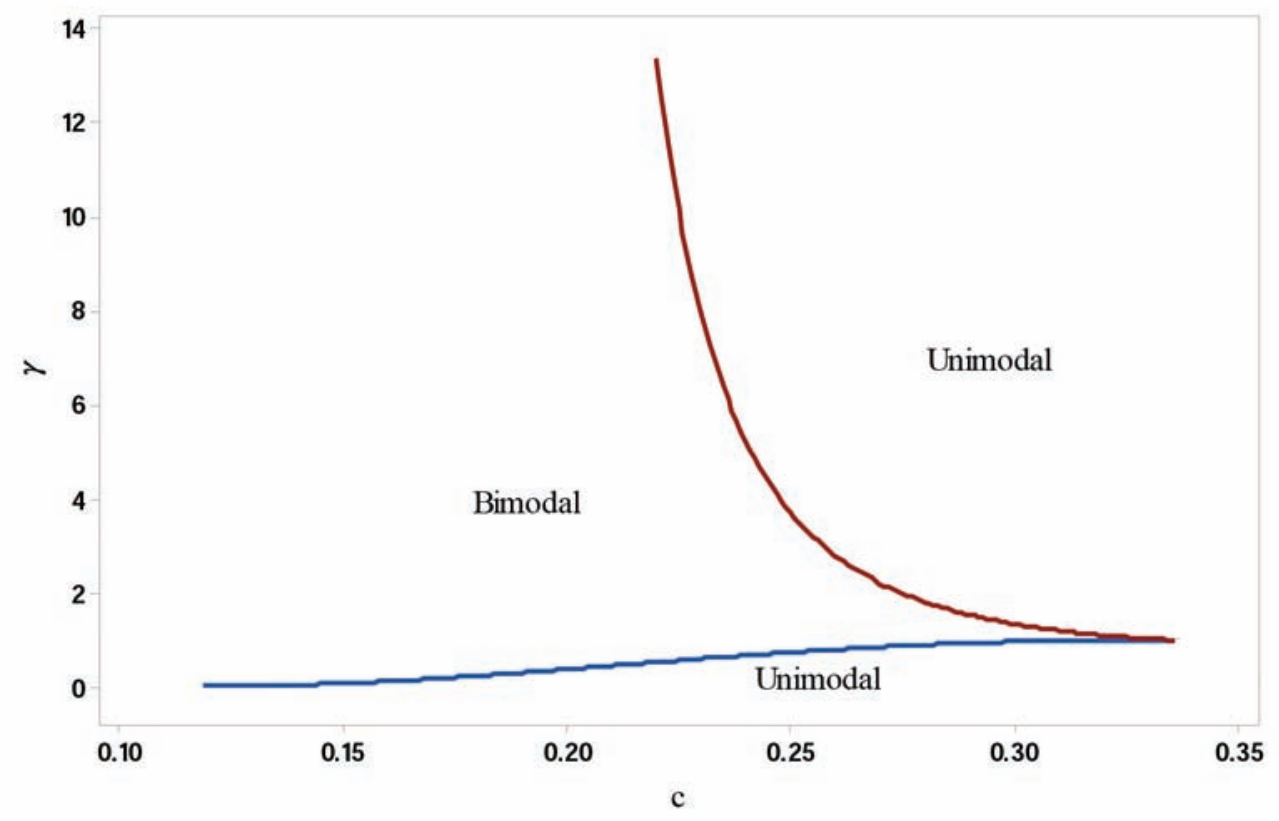

Figure 3 Unimodal and bimodal regions of $\mathrm{W}-\mathrm{N}\{\mathrm{LL}\}$ distribution.

\subsection{Shannon Entropy}

The Shannon entropy of a random variable $X$ is a measure of variation of uncertainty. It is defined by $\operatorname{Ref}$. [11] as $E\left\{-\log \left[f_{X}(x)\right]\right\}$ for a random variable with $\operatorname{PDF} f_{X}(x)$. On using the PDF in Eq. (4), we obtain

$$
E\left\{-\log \left[f_{X}(x)\right]\right\}=\log (\sigma \sqrt{2 \pi})-2 E\{\log (1+T)\}+\eta_{T}+E(X-\mu)^{2} /\left(2 \sigma^{2}\right)
$$

where $\eta_{T}$ is the Shannon entropy of Weibull distribution, which is given by $\eta_{T}=\xi(1-1 / c)+\log (\gamma / c)$, and $\xi$ is the Euler-Mascheroni constant. Thus, the Shannon entropy of the W-N $\{L L\}$ distribution is given by

$$
\eta_{X}=\log (\sigma \sqrt{2 \pi})-2 E\{\log (1+T)\}+\xi(1-1 / c)+\log (\gamma / c)+E(X-\mu)^{2} /\left(2 \sigma^{2}\right)
$$




\subsection{Mean Deviation about the Mean $\mu$ and Median $\mathbf{M}$}

The mean deviation about the population mean and population median are given by Ref. [3], and these are not provided here.

\section{ESTIMATION OF W-N\{LL\} DISTRIBUTION PARAMETERS}

Suppose we have a random sample of size $n$ from the $\mathrm{W}-\mathrm{N}\{\mathrm{LL}\}$ distribution with parameters $c, \gamma, \mu$ and $\sigma$. The likelihood function is given as

$$
L=L(c, \gamma, \mu, \sigma ; x)=\frac{c^{n}}{\gamma^{n c}} \prod_{i=1}^{n}\left[\frac{\varphi\left(x_{i}\right)}{\left[1-\Phi\left(x_{i}\right)\right]^{2}}\left(\frac{\Phi\left(x_{i}\right)}{1-\Phi\left(x_{i}\right)}\right)^{c-1} \exp \left\{-\left(\frac{\Phi\left(x_{i}\right)}{\gamma\left[1-\Phi\left(x_{i}\right)\right]}\right)^{c}\right\}\right] .
$$

On taking the logarithm of Eq. (6), we obtain

$$
\log L=\ell=\sum_{i=1}^{n}\left\{\ln \left(\frac{c}{\gamma^{c}}\right)+\ln \varphi\left(x_{i}\right)-(c+1) \ln \left[1-\Phi\left(x_{i}\right)\right]+(c-1) \ln \Phi\left(x_{i}\right)-\left(\frac{\Phi\left(x_{i}\right)}{\gamma\left[1-\Phi\left(x_{i}\right)\right]}\right)^{c}\right\} .
$$

On taking the partial derivatives of the log-likelihood with respect to the four parameters, we obtain the following likelihood equations:

$$
\begin{aligned}
& 0=\frac{\partial \ell}{\partial c}=\sum_{i=1}^{n}\left\{\frac{1}{c}-\ln \gamma-\ln \left[1-\Phi\left(x_{i}\right)\right]+\ln \Phi\left(x_{i}\right)-\left(\frac{\Phi\left(x_{i}\right)}{\gamma\left[1-\Phi\left(x_{i}\right)\right]}\right)^{c} \ln \left(\frac{\Phi\left(x_{i}\right)}{\gamma\left[1-\Phi\left(x_{i}\right)\right]}\right)\right\}, \\
& 0=\frac{\partial \ell}{\partial \gamma}=\sum_{i=1}^{n}\left\{\left(\frac{\Phi\left(x_{i}\right)}{\gamma\left[1-\Phi\left(x_{i}\right)\right]}\right)^{c}-1\right\} \frac{c}{\gamma}, \\
& 0=\frac{\partial \ell}{\partial \mu}=\sum_{i=1}^{n}\left\{\frac{x_{i}-\mu}{\sigma^{2}}+\left[\frac{c+1}{1-\Phi\left(x_{i}\right)}+\frac{c-1}{\Phi\left(x_{i}\right)}-\frac{c}{\gamma^{c}}\left(\frac{\Phi\left(x_{i}\right)}{\gamma\left[1-\Phi\left(x_{i}\right)\right]}\right)^{c-1} \frac{\partial \Phi\left(x_{i}\right)}{\partial \mu}\right]\right\}, \\
& 0=\frac{\partial \ell}{\partial \sigma}=\sum_{i=1}^{n}\left\{\frac{1}{\sigma}\left[\left(\frac{x_{i}-\mu}{\sigma}\right)^{2}-1\right]+\left[\frac{c+1}{1-\Phi\left(x_{i}\right)}+\frac{c-1}{\Phi\left(x_{i}\right)}-\frac{c}{\gamma^{c}}\left(\frac{\Phi\left(x_{i}\right)}{\gamma\left[1-\Phi\left(x_{i}\right)\right]}\right) \frac{\partial \Phi\left(x_{i}\right)}{\partial \sigma}\right]\right\},
\end{aligned}
$$

where $\frac{\partial \Phi\left(x_{i}\right)}{\partial \mu}=\frac{\partial \Phi\left(x_{i}\right)}{\partial x_{i}} \frac{\partial x_{i}}{\partial \mu}=-\varphi\left(x_{i}\right)$ and $\frac{\partial \Phi\left(x_{i}\right)}{\partial \sigma}=\frac{\partial \Phi\left(x_{i}\right)}{\partial x_{i}} \frac{\partial x_{i}}{\partial \sigma}=-\frac{\left(x_{i}-\mu\right) \varphi\left(x_{i}\right)}{\sigma}$.

One can obtain the second partial derivatives and these can be used to find the variance-covariance matrix for the parameter estimates. The equations in (7) through (10) are solved by an iterative technique to obtain the maximum likelihood estimates $\hat{c}, \hat{\gamma}, \hat{\mu}$, and $\hat{\sigma}$ for the parameters $c, \gamma, \mu$ and $\sigma$ respectively. To find the initial estimates for the parameters we first consider the random sample as a sample from the normal distribution. We estimate $\mu$ and $\sigma$ by using the sample mean $\bar{x}$ and sample standard deviation $s$. Second, we transform the W-N $\{L L\}$ data to that of a Weibull random sample by using Lemma 1 to obtain $y_{i}=\Phi\left(x_{i}\right) /\left[1-\Phi\left(x_{i}\right)\right]$, where the $x_{i}$ has mean $\bar{x}$ and standard deviation $s$. By using the moment estimates for the Weibull parameters $c$ and $\gamma$, we obtain the initial estimates for parameters $c$ and $\gamma$. These estimates are given by $c_{*}=\pi /\left[6 s_{\log \left(y_{i}\right)}\right]$ and $\gamma_{*}=\exp \left[-\bar{y}_{\log \left(y_{i}\right)}-\delta / c_{*}\right]$, where $s_{\log \left(y_{i}\right)}$ and $\bar{y}_{\log \left(y_{i}\right)}$ are the sample standard deviation and the sample mean for $\log \left(y_{i}\right)$ and $\delta$ is the Euler gamma constant (Ref. [12], pp. 642-643).

\section{SIMULATION}

In order to evaluate the performance of the maximum likelihood estimation method, we conduct a simulation study. The quantile function in Eq. (5) is used to generate a random sample from the Weibull-normal $\{\log$-logistic $\}$ distribution with parameters $c, \gamma, \mu$ and $\sigma$. The parameters $\mu$ and $\sigma$ are fixed at $\mu=4$ and $\sigma=2$ for the whole simulation study. The parameter combinations for $c$ and $\gamma$ are varied and they are given in Table 1. The combinations are selected to reflect when the distribution is bimodal and unimodal. The case where $c=0.2$ and $\gamma=2,4$, and 6 represent bimodal cases while $c=0.5$ and $\gamma=2,4$, and 6 represent unimodal cases. For each parameter combination and each sample size, the simulation process is repeated 200 times. The average bias (actual-estimate) and the standard deviation of the parameter estimates are reported in Table 1 . The biases are relatively small when compared to the standard deviations. In most cases, as the sample size increases, the standard deviations of the estimators decrease. 
Table 1 Bias and standard deviation of maximum likelihood estimates when $\mu=4$ and $\sigma=2$.

\begin{tabular}{ccccccc}
\hline & \multicolumn{2}{c}{ Actual values } & \multicolumn{3}{c}{ Bias with standard deviation in parentheses } \\
$\boldsymbol{n}$ & $\boldsymbol{c}$ & $\gamma$ & $\hat{\boldsymbol{c}}$ & $\hat{\gamma}$ & $\hat{\boldsymbol{\mu}}$ & $\hat{\sigma}$ \\
\hline & 0.2 & 2.0 & $-0.006(0.036)$ & $0.062(0.434)$ & $-0.018(0.268)$ & $-0.036(0.235)$ \\
& 0.2 & 4.0 & $-0.006(0.037)$ & $0.168(0.920)$ & $-0.029(0.253)$ & $-0.021(0.235)$ \\
& 0.2 & 6.0 & $0.003(0.035)$ & $0.486(1.292)$ & $0.012(0.301)$ & $0.023(0.227)$ \\
& 0.5 & 2.0 & $0.009(0.102)$ & $0.077(0.428)$ & $-0.060(0.263)$ & $0.033(0.335)$ \\
& 0.5 & 4.0 & $0.020(0.092)$ & $0.045(0.895)$ & $-0.098(0.311)$ & $0.075(0.282)$ \\
& 0.5 & 6.0 & $0.006(0.094)$ & $0.073(1.333)$ & $-0.081(0.384)$ & $0.040(0.294)$ \\
\hline & 0.2 & 2.0 & $-0.004(0.032)$ & $0.043(0.435)$ & $-0.015(0.208)$ & $-0.019(0.209)$ \\
& 0.2 & 4.0 & $-0.002(0.030)$ & $0.204(0.855)$ & $-0.042(0.208)$ & $-0.007(0.186)$ \\
& 0.2 & 6.0 & $0.001(0.030)$ & $0.428(1.275)$ & $-0.013(0.199)$ & $0.012(0.191)$ \\
& 0.5 & 2.0 & $0.002(0.088)$ & $0.046(0.412)$ & $-0.059(0.230)$ & $0.011(0.282)$ \\
& 0.5 & 4.0 & $-0.005(0.084)$ & $0.093(0.892)$ & $-0.052(0.283)$ & $-0.011(0.273)$ \\
& 0.5 & 6.0 & $-0.003(0.085)$ & $0.148(1.388)$ & $-0.047(0.300)$ & $-0.006(0.263)$ \\
\hline & 0.2 & 2.0 & $-0.004(0.030)$ & $0.073(0.410)$ & $-0.031(0.181)$ & $-0.021(0.197)$ \\
& 0.2 & 4.0 & $-0.001(0.027)$ & $0.099(0.854)$ & $-0.035(0.194)$ & $0.002(0.169)$ \\
& 0.2 & 6.0 & $-0.003(0.028)$ & $0.544(1.317)$ & $-0.033(0.182)$ & $-0.015(0.177)$ \\
& 0.5 & 2.0 & $-0.009(0.081)$ & $0.059(0.389)$ & $-0.058(0.225)$ & $-0.024(0.259)$ \\
& 0.5 & 4.0 & $0.003(0.074)$ & $0.032(0.821)$ & $-0.038(0.226)$ & $0.009(0.229)$ \\
& 0.5 & 6.0 & $-0.010(0.070)$ & $0.183(1.243)$ & $-0.044(0.238)$ & $-0.020(0.215)$ \\
\hline
\end{tabular}

\section{APPLICATIONS}

In this section, the $\mathrm{W}-\mathrm{N}\{\mathrm{LL}\}$ distribution is applied to three data sets and the results are compared with other distributions.

\subsection{Amount of Money Paid to Insurance Policy Holders}

The data analyzed in this sub-section is the amount of money paid by an insurance company in Nigeria to policy holders when they discontinue and 'drop out'. The data consists of a random sample of 195 customers and the data is available from the second author. The logarithm of the amount paid is fitted by using the Weibull-normal\{log-logistic $\}$ distribution, the BN distribution defined and studied by Ref. [4], and Cauchy-Weibull\{logistic $\}(\mathrm{C}-\mathrm{W}\{\mathrm{L}\})$ distribution defined and studied by Ref. [13]. The data is skewed to the right (skewness = 0.2574 and kurtosis $=-0.5231$ ). The results from the three distributions are presented in Table 2. The Akaike Information Criterion (AIC) and the Kolmogorov-Smirnov (KS) goodness of fit statistic with its corresponding $p$-value are reported in Table 2 . From the table, both the $\mathrm{W}-\mathrm{N}\{\mathrm{LL}\}$ and $\mathrm{BN}$ distributions provide adequate fit to the data set while the $\mathrm{C}-\mathrm{W}\{\mathrm{L}\}$ distribution does not provide a good fit to the data.

Table 2 Parameter estimates (standard errors) for insurance policy holders data.

\begin{tabular}{|c|c|c|c|}
\hline Distribution & $\mathbf{C}-\mathbf{W}\{\mathbf{L}\}$ & BN & $\mathbf{W}-\mathbf{N}\{\mathbf{L L}\}$ \\
\hline \multirow[t]{4}{*}{ Parameter estimates } & $\hat{\alpha}=-3.7319(1.0491)$ & $\hat{\mu}=1.4351(0.6820)$ & $\hat{c}=0.2187(0.0661)$ \\
\hline & $\hat{\beta}=2.742(0.4851)$ & $\hat{\sigma}=0.1634(0.0064)$ & $\hat{\gamma}=0.0539(0.0540)$ \\
\hline & $\hat{c}=31.6786(5.4520)$ & $\hat{c}=10.0665(0.3806)$ & $\hat{\mu}=13.688(0.2749)$ \\
\hline & $\hat{\gamma}=13.2296(0.2127)$ & $\hat{\gamma}=0.7949(0.0686)$ & $\hat{\sigma}=0.7350(0.1520)$ \\
\hline Log-likelihood & -364.00 & -341.63 & -341.75 \\
\hline AIC & 736.0 & 691.3 & 691.5 \\
\hline KS statistic ( $p$-value) & $0.1225(0.0057)$ & $0.0480(0.7606)$ & $0.0504(0.7042)$ \\
\hline
\end{tabular}

\subsection{USS Halfbeak Engine Data}

The USS Halfbeak diesel engine data was used by Ref. [14] to illustrate the application of McDonald-gamma (Mc-G) distribution. The distribution of the data is highly skewed to the left and it is platykurtic (skewness $=-1.5764$ and kurtosis $=1.6525$ ). Ref. [2] also fitted the data to a four-parameter normal-Weibull \{Cauchy $(\mathrm{N}-\mathrm{W}\{\mathrm{C}\})$ distribution. The fits from $\mathrm{N}-\mathrm{W}\{\mathrm{C}\}$ and Mc-G distributions are obtained from Ref. [2] and are given in Table 3. In Table 3, we provide the parameter estimates and the goodness of fit statistics. Only two of the three distributions in Table 3 provide adequate fit to the data set. Furthermore, both $\mathrm{N}-\mathrm{W}\{\mathrm{C}\}$ and $\mathrm{W}-\mathrm{N}\{\mathrm{LL}\}$ provide almost identical fit to the data when we consider both the log-likelihood and the AIC statistics. 
Table 3 Parameter estimates (standard errors) for the USS Halfbeak diesel engine data.

\begin{tabular}{|c|c|c|c|}
\hline Distribution & Mc_G* & $\mathbf{N}-\mathbf{W}\{\mathbf{C}\}^{*}$ & $\mathbf{W}-\mathbf{N}\{\mathbf{L L}\}$ \\
\hline & $\hat{\beta}=2.030(0.022)$ & $\hat{\sigma}=4.217(1.207)$ & $\hat{\gamma}=13707(0.0167)$ \\
\hline & $\hat{b}=200.04(60.178)$ & $\hat{\gamma}=10.073(0.936)$ & $\hat{\sigma}=2.2437(0.3186)$ \\
\hline & $\hat{c}=0.2796(0.014)$ & & \\
\hline Log-likelihood & -217.35 & -196.45 & -196.18 \\
\hline
\end{tabular}

* Estimates from Ref. [2]

\subsection{Crops Produce Data}

The data analyzed in this sub-section are the prices of some crops produced in Nigeria. The variable is the farmgate price in Naira (Nigeria monetary unit) per kilogram of crops obtained from Nigeria National Bureau of Statistics. The variable is the addition of farmgate prices for the following 13 crops produce: cowpea, cassava, maize, cocoyam, groundnut, rice, yam, rice, melon, soybeans, sesame seed and palm oil. The data was collected for 2006 through 2015 for each of the 36 states in Nigeria and the Federal Capital Territory (Abuja) leading to $n=37$ data points for each year. For this analysis, we used the data for 2015, the most recent year that has data available (the data is available from the third author). The data is negatively skewed (skewness $=-0.3010$ and kurtosis $=-0.7214$ )

We applied the four-parameter BN distribution by Ref. [4], the four-parameter normal-Weibull $\{$ Cauchy $\}$ (N-W $\{\mathrm{C}\})$ distribution by Ref. [2] and the $\mathrm{W}-\mathrm{N}\{\mathrm{LL}\}$ distribution defined and studied in this paper to analyze the data. The results of the fits are provided in Table 4 . All the three distributions provide adequate fit to the data. However, the W-N $\{L L\}$ distribution provides the best K-S statistic and has a slightly smaller AIC value than the other two distributions.

Table 4 Parameter estimates (standard errors) for the crops produce data.

\begin{tabular}{|c|c|c|c|}
\hline Distribution & BN & $\mathbf{N}-\mathbf{W}\{\mathbf{C}\}$ & $\mathbf{W}-\mathbf{N}\{\mathbf{L L}\}$ \\
\hline & $\hat{\beta}=0.0602(0.0157)$ & $\hat{\sigma}=1.0787(0.4402)$ & $\hat{\gamma}=3.6384(8.9261)$ \\
\hline & $\hat{\sigma}=4.6597(0.5763)$ & $\hat{\gamma}=222.36(6.8605)$ & $\hat{\sigma}=9.5455(5.2492)$ \\
\hline Log-likelihood & -180.06 & -180.38 & -179.36 \\
\hline $\mathrm{AIC}$ & 368.1 & 368.8 & 366.7 \\
\hline
\end{tabular}

\section{SUMMARY}

A new generalization of the normal distribution is defined and studied. The Weibull-normal $\{\log -\operatorname{logistic}\}$ (W-N $\{L \mathrm{~L}\})$ distribution can be skewed to the left or skewed to the right. The distribution can be unimodal or bimodal. When the shape parameter $c=1$, the $\mathrm{W}-\mathrm{N}\{\mathrm{LL}\}$ distribution reduces to the exponential-normal\{log-logistic $\}$ distribution with three parameters. This special case is always unimodal since the shape parameter $c$ is set to 1 . The method of maximum likelihood estimation is proposed for estimating the parameters of $\mathrm{W}$ - $\mathrm{N}\{\mathrm{LL}\}$ distribution. The distribution is applied to three data sets and it is found to perform well in fitting the data sets compared to other distributions. From the data sets in Section 6, we notice that the W-N $\{L L\}$ distribution is capable of providing adequate fit to data sets that are about symmetric or skewed to the left or skewed to the right.

\section{ACKNOWLEDGEMENT}

The first author gratefully acknowledges the financial support received from the U.S. Department of State, Bureau of Education and Cultural Affairs under the Fulbright Grant \# PS00230565. 


\section{REFERENCES}

1. C. Lee, F. Famoye, A. Alzaatreh, WIREs Comp. Stat. 5 (2013), 219-238.

2. M. Aljarrah, C. Lee, F. Famoye. J. Stat. Distrib. Appl. 1 (2014), 2.

3. A. Alzaatreh, C. Lee, F. Famoye. J. Stat. Distrib. Appl. 1 (2014), 16.

4. N. Eugene, C. Lee, F. Famoye, Commun. Stat. Theory Methods. 31(4) (2002), 497-512.

5. F. Famoye, C. Lee, N. Eugene. J. Mod. Appl. Stat. Methods. 3 (2004), 85-103.

6. G.M. Cordeiro, M. de Castro. J. Stat. Comput. Simulat. 81 (2011), 883-898.

7. M.C. Jones, Stat. Methodol. 6 (2009), 70-81.

8. P. Kumaraswamy, J. Hydrol. 46 (1980), 79-88.

9. C. Alexander, G.M. Cordeiro, E.M.M. Ortega, J.M. Sarabia, Comput. Stat. Data Anal. 56 (2012), 1880-1897.

10. A. Alzaatreh, C. Lee, F. Famoye. Metron. 71 (2013), 63-79.

11. C.E. Shannon, Bell Syst. Tech. J. 27 (1948), 379-432.

12. N.L. Johnson, S. Kotz, N. Balakrishnan, Continuous Univariate Distributions, second ed., John Wiley and Sons, Inc., New York, 1994.

13. M. Almheidat, F. Famoye, C. Lee, Int. J. Stat. Prob. 4(3) (2015), 18-35.

14. F.W.P. Marciano, A.D.C. Nascimento, M. Santos-Neto, G.M. Cordeiro, Int. J. Stat. Prob. 1(1) (2012), 53-71. 